\title{
Associação entre adesão ao tratamento anti-hipertensivo e integralidade no atendimento de enfermeiros
}

\author{
Association between antihypertensive treatment adherence and comprehensive nursing care \\ Asociación entre adhesión al tratamiento antihipertensivo e integralidad en la atención de los enfermeros
}

\author{
Antônia Sylca de Jesus Sousa'; Thereza Maria Magalhães Moreira"; \\ Ana Larissa Gomes Machado"l'; Ana Zaira da Silva ${ }^{\prime V}$
}

\begin{abstract}
RESUMO
Objetivo: verificar a associação entre nível de adesão dos clientes ao tratamento anti-hipertensivo e a integralidade no atendimento de enfermeiros. Método: pesquisa de abordagem quantitativa, desenvolvida na cidade de Fortaleza-CE, com 602 hipertensos atendidos em um Centro de Saúde de Atenção Secundária. Para identificar a presença ou não da adesão, aplicou-se o Teste de Morisky-Green e a presença da integralidade na assistência foi obtida por um check-list baseado nos pressupostos da Starfield (acessibilidade, acolhimento, vínculo e enfoque familiar). Foi realizado um entrecruzamento das variáveis por meio do teste estatístico Quiquadrado de Pearson (X2). O presente estudo foi aprovado pelo Comitê de Ética em Pesquisa. Resultados: não houve associação entre adesão e integralidade no atendimento de enfermeiros $(p=0,758)$. Conclusão: verifica-se a necessidade de se realizar um cuidado de enfermagem centrado na integralidade da assistência, de forma a buscar cada vez a adesão dos pacientes hipertensos ao tratamento. Descritores: Integralidade em saúde; assistência integral à saúde; adesão à medicação; enfermagem.
\end{abstract}

\section{ABSTRACT}

Objective: to examine the association between client adherence to antihypertensive treatment and comprehensive nursing care. Methodology: quantitative study conducted Fortaleza, Ceará, with 602 hypertensive patients treated at a Secondary Health Care Center. Treatment adherence was identified by applying Morisky-Green test and the comprehensiveness of care was assessed using a checklist based on Starfield's principles (accessibility, placement, involvement and family approach). Variables were correlated using Pearson's Chi-Square Test (X2). The study was approved by the research ethics committee. Results: no association was found between treatment adherence and the comprehensiveness of nursing care $(p=0.758)$. Conclusion: there was found to be a need to provide nursing care centered on comprehensiveness, with a view always to improving treatment adherence among hypertensive patients. Descriptors: Integrality in health; comprehensive health care; medication adherence; nursing.

\section{RESUMEN}

Objetivo: verificar la asociación entre el nivel de adhesión de los clientes al tratamiento antihipertensivo y la integralidad en la atención de los enfermeros. Método: investigación de enfoque cuantitativo, desarrollada en la ciudad de Fortaleza-Ceará, junto a 602 pacientes hipertensos atendidos en un Centro de Salud de Atención Secundaria. Para identificar la presencia o no de la adhesión, se aplicó el Test de Morisky-Green y la presencia de la integralidad en la asistencia se obtuvo a través de una check-list basada en los principios de Starfield (accesibilidad, acogimiento, vínculo y enfoque familiar). Se realizó un entrecruce de las variables a través del test estadístico Chi Cuadrado de Pearson (X2). El presente estudio fue aprobado por el Comité de Ética en Investigación. Resultados: no hubo asociación entre adhesión e integralidad en la atención de los enfermeros $(p=0,758)$. Conclusión: se verificó la necesidad de desarrollar un cuidado de enfermería focalizado en la integralidad de la asistencia, con el objeto de lograr una mayor adhesión de pacientes hipertensos al tratamiento.

Descriptores: Integralidad en salud; atención integral de salud; cumplimiento de la medicación; enfermería.

\section{INTRODUÇÃo}

Conceituadas como um conjunto de enfermidades que apresentam altas taxas de mortalidade, morbidade e incapacidade, as Doenças Crônicas Não Transmissíveis (DCNT) constituem-se um problema de saúde pública que se perpetua ao longo dos anos, especialmente nos países em desenvolvimento, como o Brasil. Dentre as mais importantes DCNT destaca-se a Hipertensão Arterial Sistêmica (HAS) por apresentar elevada incidência e prevalência nas sociedades contemporâneas, constituindo-se no principal motivo de consultas na atenção primária, assim como nos demais níveis de atenção à saúde $\mathrm{e}^{1-3}$. 
O tratamento farmacológico aliado a medidas não farmacológicas favorece o controle da pressão arterial (PA). No entanto, evidências científicas têm revelado que a falta de adesão ao tratamento é um dos principais fatores que dificultam o efetivo controle da HAS, pois cerca de $40 \%$ a $60 \%$ dos pacientes não fazem uso da medicação prescrita, e essa porcentagem aumenta quando a falta de adesão se relaciona ao estilo de vida, fato convergente com os resultados de pesquisas recentes que apontam que apenas metade das pessoas com HAS conseguem manter os níveis tensionais ${ }^{4-6}$.

Enquanto categoria profissional diretamente ligada ao cuidado e, portanto, muito próxima ao cliente em todos os níveis de atenção do sistema, a enfermagem tem papel fundamental, especialmente no que diz respeito à implementação da integralidade do cuidado ${ }^{7}$. Por representar uma das três diretrizes do Sistema Único de Saúde-SUS contempladas na Constituição Federal de 1988, a integralidade se constitui num meio eficaz de obter excelência nos resultados, pelo desenvolvimento de ações que tem como base, dentre outros instrumentos, a educação em saúde, desenvolvida nos diversos cenários de atuação dos enfermeiros.

Tendo em vista a influência que o cuidado dispensando pelos enfermeiros, pautado especialmente na integralidade, exerce sobre a vida e saúde das pessoas, objetivou-se verificar a associação entre nível de adesão dos clientes ao tratamento anti-hipertensivo e a integralidade no atendimento de enfermeiros.

\section{REVISÃo DE LITERATURA}

A adesão ao tratamento relaciona-se diretamente com a aceitação e o reconhecimento da doença, neste caso a HAS, pois a sua terapêutica ao tratamento na situação de adoecimento crônico não perpassa somente pela motivação pessoal do paciente. Diferentes nuances interferem nesse processo, tais como a baixa condição socioeconômica, a deficiência de autocuidado e de conhecimentos sobre a enfermidade, entre outros ${ }^{8}$. Assim, para se realizar a adesão ao tratamento é preciso que o comportamento de cada sujeito esteja de acordo com as orientações estabelecidas pelos profissionais de saúde, estando estas intimamente ligadas à terapia medicamentosa e às mudanças no estilo de vida ${ }^{5,9,10}$.

Nesse contexto, ressalta-se que a efetiva participação dos profissionais da saúde faz-se necessária para o alcance da adesão ao tratamento anti-hipertensivo. 0 enfermeiro, enquanto integrante da equipe de saúde, assume a corresponsabilidade das ações do cuidado para a promoção da saúde e prevenção de agravos na vida e saúde dos sujeitos, principalmente quando estes precisam viver com algum tipo de doença crônica. Por meio do conhecimento científico e de seu papel de educador, esse profissional tem a possibilidade de conduzir os pacientes para a realização de um tratamento satisfatório, o que resultará na melhoria do seu bem-estar ${ }^{11,12}$.
A integralidade na saúde vai além de uma diretriz, onde uma de suas dimensões é a observância do conceito ampliado em saúde, que deve dar atenção não só aos aspectos de doença, mas também aos sociais e psicológicos. Ela também emerge como um princípio de organização contínua do processo de trabalho, pela ampliação de possibilidades que buscam apreender as necessidades de saúde de um dado grupo populacional. Dessa forma, falar em integralidade é falar também de interdisciplinaridade do cuidado, é remeter à realização de práticas intersubjetivas por meio do estabelecimento de relações interpessoais entre os sujeitos, pelas quais se alcança o sucesso com práticas de saúde dialógicas, principalmente quando estas se estabelecem entre enfermeiros e as pessoas que convivem com a HAS, pois nesse caso o convívio é mais intenso e estreito ${ }^{13-15}$.

\section{Metodologia}

Trata-se de estudo de natureza analítica, com abordagem quantitativa, realizado em um centro de referência de Fortaleza-CE, com coleta de dados no período de abril a junho de 2013. A população do estudo constituiu-se por hipertensos atendidos na unidade e foi calculada pela fórmula para população infinita ${ }^{16}$, sendo de 602 hipertensos.

Para analisar a presença ou não de adesão, foi aplicado o Teste de Morisky e Green ${ }^{17}$, composto por quatro perguntas: Você às vezes tem problemas em se lembrar de tomar a sua medicação? Você às vezes se descuida de tomar seu medicamento? Quando está se sentindo bem, você às vezes para de tomar seu medicamento? Às vezes, se você se sentir pior ao tomar a medicação, você para de tomá-la?

Dessa forma, o paciente foi classificado no grupo de adesão, quando as respostas a todas as perguntas foram negativas. Porém, quando pelo menos uma das respostas foi afirmativa, o paciente foi classificado com não adesão ${ }^{18}$.

Em seguida, foi verificado como se configurava a integralidade do atendimento. Para obtenção deste resultado, foi avaliada a integralidade baseada nos dispositivos propostos pela Starfield ${ }^{19}$, operacionalizados como integralidade horizontal (acesso, acolhimento, vínculo e enfoque familiar) ${ }^{19,20}$.

Os dispositivos da integralidade, o conceito apresentado e a reta de análise utilizada para atribuição de uma nota ao dispositivo, conforme a visão do usuário, estão descritos na Figura 1.

A reta de análise propõe notas para os dispositivos da integralidade, nos quais os participantes deram um valor a partir da visão que possuem a respeito do atendimento recebido no serviço. Isto foi feito por meio de um instrumento check-list com escala do tipo Likert, com pontuação de zero a dez (0---1---2---3---4--5---6---7---8---9---10). A partir daí, buscou-se classificar a presença da integralidade quando se obteve nota 


\begin{tabular}{|c|c|c|}
\hline Dispositivos & Conceito & $\begin{array}{c}\text { Reta de análise (nota atribuída aos } \\
\text { pressupostos) }\end{array}$ \\
\hline $\begin{array}{l}\text { Acessibili- } \\
\text { dade }\end{array}$ & $\begin{array}{l}\text { Fácil acesso aos serviços de saúde, oferecido na instituição } \\
\text { (com horários e dias de atendimento, facilidade de se } \\
\text { consultar e obter medicamentos). }\end{array}$ & 0---1---2---3----4---5---6---7---8---9---10 \\
\hline Acolhimento & $\begin{array}{l}\text { Estabelecimento de interações, por meio de escuta } \\
\text { qualificada, buscando agir em função do outro/reconhecer } \\
\text { sua biografia e a estória do seu adoecimento. }\end{array}$ & 0---1---2---3---4---5---6---7---8---9---10 \\
\hline Vínculo & Qualidade das relações entre os profissionais e a população. & 0---1---2---3----4---5---6----7---8---9---10 \\
\hline $\begin{array}{l}\text { Enfoque } \\
\text { familiar }\end{array}$ & $\begin{array}{l}\text { Consideração da família e do ambiente familiar nas } \\
\text { consultas do serviço. }\end{array}$ & 0---1---2---3---4----5---6---7---8---9---10 \\
\hline
\end{tabular}

FIGURA 1: Dispositivos da integralidade, seus conceitos e reta de análise. Fortaleza-CE, 2013.

sete em $50 \%$ dos dispositivos, na pontuação total de cada questionário. Do contrário, considerou-se a ausência de integralidade.

De posse dos resultados da presença da adesão/ não adesão ao tratamento anti-hipertensivo e da averiguação da presença/ausência de integralidade na assistência de enfermagem aos pacientes, verificou-se se existia associação entre estas variáveis. Para tanto, fez-se o entrecruzamento de variáveis por meio do teste estatístico Quiquadrado de Pearson (X2). Os dados coletados foram tabulados e organizados utilizando o Software Statistical Package for Social Sciences (SPSS) versão 20.0. Após serem agrupados, os resultados foram apresentados em figura e tabela, sendo realizada a discussão de acordo com a literatura pertinente.

O projeto foi aprovado pelo Comitê de Ética em Pesquisa com seres humanos da Universidade Estadual do Ceará, com parecer $n^{\circ} 400.990$.

\section{RESULTADOS E DISCUSSÃo}

A associação entre adesão ao tratamento anti-hipertensivo e integralidade no atendimento pelo enfermeiro, pode ser observado na Tabela 1:

TABELA 1: Análise da adesão ao tratamento anti-hipertensivo segundo presença e ausência de integralidade da assistência. Fortaleza-Ceará-Brasil, 2013.

\begin{tabular}{lcccccc}
\hline \multirow{2}{*}{ Integralidade } & \multicolumn{2}{c}{ Adesão } \\
& \multicolumn{2}{c}{ Sim } & \multicolumn{2}{c}{ Não } & \multicolumn{2}{c}{ TOTAL } \\
& $\mathbf{n}$ & $\mathbf{\%}$ & $\mathbf{n}$ & $\mathbf{\%}$ & $\mathbf{n}$ & $\mathbf{\%}$ \\
\hline Sim & 320 & 86,7 & 200 & 85,8 & 520 & 86,4 \\
Não & 49 & 13,3 & 33 & 14,2 & 82 & 13,6 \\
TOTAL & 369 & 61,3 & 233 & 38,7 & 602 & 100 \\
\hline
\end{tabular}

Verifica-se que dos 369 (61,3\%) que aderiram ao tratamento, $320(86,7 \%)$ referiram presença da integralidade na assistência. Já dos $233(38,7 \%)$ pacientes que não aderiram ao tratamento, 200 (85,8\%) deles também constatou presença da integralidade.
Ao entrecruzar adesão e integralidade do cuidado, constatou-se que quase dois terços dos pacientes aderiam ao tratamento da HAS, sendo a integralidade constatada por pacientes com e sem adesão ao tratamento; podendo-se afirmar desse modo, que não houve associação entre adesão e integralidade no tratamento anti-hipertensivo, pois não foi encontrada associação estatisticamente significativa $(p=0,758)$.

Viu-se, ainda, que a adesão foi $1 \%$ superior nos pacientes que constataram integralidade. Isto pode refletir baixa acurácia na constatação da presença ou não de integralidade, decorrente do critério adotado para constatar presença de integralidade (obtenção de nota sete em cinquenta por cento dos dispositivos) ou dificuldade das pessoas para atribuíram notas ao seu atendimento.

Ressalte-se que o instrumento avaliativo da integralidade tinha sete notas para avaliação negativa (0-6) e apenas quatro para positivas (7-10) e que, ainda assim apenas 82 pacientes $(13,6 \%)$ ficaram na categoria de ausência de integralidade.

Em estudo realizado para avaliar a adesão ao tratamento medicamentoso em usuários cadastrados no Programa Hiperdia no município de Teresina - Piauí (PI), os autores utilizaram o teste de Morisky-Green para avaliar a adesão e não adesão ao tratamento, teste este utilizado também nesta pesquisa, e constataram que quase metade dos participantes não esqueciam de utilizar o fármaco ${ }^{21}$.

Entre os fatores que influenciam para a pouca adesão, pesquisas comprovam que os fatores socioeconômicos se destacam com elevada frequência, pois, quanto mais baixos seus níveis, menores as taxas de adesão ao tratamento anti-hipertensivo, tendo em vista que o conhecimento da doença é mais superficial e, difícil o acesso aos serviços de saúde ${ }^{9,22}$.

Uma boa estratégia para estimular o paciente aderir ao tratamento é conscientizá-lo dos malefícios que a hipertensão arterial pode resultar quando não tratada adequadamente. Além dos riscos inerentes ao tratamento, seus benefícios e peculiaridades devem ser evidenciados, favorecendo que o indivíduo se torne um sujeito ativo no seu próprio tratamento ${ }^{23,24}$. 
Ao averiguar a presença da integralidade na assistência de enfermagem aos pacientes com hipertensão, confirmou-se serem muitos os sentidos da integralidade, incluindo entre eles o direito universal ao atendimento das necessidades de saúde do usuário, buscando resolutividade nas ações, e respostas adequadas aos problemas identificados. A integralidade busca atender aos aspectos orgânicos, emocionais, sociais e espirituais envoltos no adoecimento das pessoas dentro de uma contextualização social, visando a restauração da vitalidade dos indivíduos e coletividades ${ }^{7}$.

No que diz respeito ao acolhimento, este consiste em uma ferramenta que permite o acesso universal aos serviços, tornando-os humanizados, integrais, com resolutividade e qualidade fazendo com que os problemas de saúde dos usuários se tornem responsabilidade dos profissionais. Acolher bem é uma estratégia fundamental para o estabelecimento de vínculo entre equipe de saúde e usuários, sendo considerado um instrumento de trabalho capaz de produzir resultados satisfatórios em intervalos reduzidos ${ }^{5,25}$

Assim como o estabelecimento de vínculos, o acolhimento constitui-se ferramenta de primordial significância no contexto de profissionais da saúde junto a pessoas com algum tipo de doença crônica, como é o caso da hipertensão. Ambos fazem parte dos objetivos estabelecidos pela Política Nacional de Humanização, através da ampliação do acesso e da valorização do usuário enquanto ator principal do processo de saúde-doença. A partir do vínculo é possível o estabelecimento de afeto, entre profissionais e pacientes, mediante o qual será possível o desenvolvimento de relações democráticas com foco no cuidado prestado ${ }^{26}$.

Sobre o Enfoque Familiar, tem-se que a não adesão ao tratamento medicamentoso de uma doença crônica envolve muito mais que os conflitos relativos à doença. Esta é uma questão complexa que abrange, dentre outros aspectos, a inexistência de apoio familiar, configurando-se este de fundamental importância para o enfrentamento da condição de saúde. O apoio se manifesta de várias maneiras, tais como: lembrar o hipertenso do horário das medicações e de orientá-lo na dieta; ou até mesmo na disposição para acompanhar o hipertenso às consultas ${ }^{27}$.

Com efeito, o cuidado de enfermagem envolve mais do que um simples fazer, envolve compromisso, responsabilidade, conhecimento, vínculo e acolhimento. Ressalta-se que o estabelecimento de uma relação adequada entre profissional e usuário, o diálogo/comunicação, o conforto dos acompanhantes, uma assistência com enfoque na segurança do paciente e a integralidade na saúde mantida em ambientes intra e extra-hospitalar contribuem para a humanização da assistência, pois o cuidar pressupõe além do saber técnico, um olhar amplo que possibilita conhecer o ser humano em sua totalidade, relacionar-se $\mathrm{s}^{28,29}$.
É oportuno destacar assim, a necessidade de um atendimento mais rigoroso e individualizado aos pacientes com hipertensão, através de uma equipe multidisciplinar que busque avalia-los de forma holística, abrangendo todo o contexto político, social em que estão inseridos.

\section{CONCLUSÃo}

Constatou-se, a partir da utilização do Teste de Morisky-Green, o predomínio da adesão ao tratamento da HAS entre os participantes deste estudo. Todavia, ao entrecruzar adesão e integralidade do cuidado, não houve associação estatística.

Assim, conclui-se que manter a integralidade no cotidiano das práticas de saúde é defender que a oferta de cuidados esteja inserida adequadamente no contexto particular de cada momento. Ressalta-se que qualquer perspectiva de mudança que vise à melhoria da qualidade da assistência de enfermagem deve apostar em processos de interação entre usuários e profissionais, identificando obstáculos e potencialidades mediante experiências e vivências, que objetivem cuidados amplos e pertinentes às mais variadas realidades.

É notória a necessidade do estabelecimento de vínculos nas relações entre profissionais e usuários, constituindo-se este no grande desafio da integralidade do cuidado; assim como da produção de ações de promoção da saúde visando à (Re) construção do cuidado centrado no sujeito.

É preciso promover um cuidado de enfermagem centrado na integralidade da assistência, que busque cada vez mais a adesão dos sujeitos ao tratamento, através de um ambiente que favoreça a acessibilidade $e$ o acolhimento, estando os dispositivos da integralidade envoltos numa ampla rede de relações profissionais cujo centro das ações deve ser o cliente e suas necessidades de saúde.

Como limitação do estudo enfatiza-se a falta de instrumentos específicos para medida da integralidade no atendimento dos enfermeiros, assim, os escores propostos para classificar a presença da integralidade não apresentaram pontos de corte pré-definidos na literatura. Ainda, há escassa literatura acerca da integralidade no atendimento do enfermeiro.

\section{REFERÊNCIAS}

1. Martins LN, Souza LS, Silva CF, Machado RS, Silva CEF, Vilagra $\mathrm{MM}$, et al. Fatores de risco cardiovascular em adultos admitidos na unidade de dor torácica em Vassouras, RJ. Rev. Bras. Cardiol. 2011; 24(5):299-307.

2. Ministério da Saúde (Br). Cadernos de Atenção Básica: Estratégias para o cuidado da pessoa com doença crônica - Hipertensão Arterial Sistêmica. Brasília (DF): Ministério da Saúde; 2013.

3. Santos MVR, Oliveira DC, Arraes LB, Oliveira DAGC, Medeiros L, Novaes MA. Adesão ao tratamento anti-hipertensivo: conceitos, aferição estratégias inovadoras de abordagem. Rev. Bras. Clin. Med. 2013; 11(1):55-61.

4. Motter FR, Olinto MTA, Paniz VMV. Avaliação do conhecimento 
sobre níveis tensionais e cronicidade da hipertensão: estudo com usuários de uma Farmácia Básica no sul do Brasil. Cad. Saúde Pública (Online). 2015; 31(2):395-404.

5. Dougados M, Soubrier M, Perrodeau E, Gossec L, Fayet F, Gilson $M$, et al. Impact of a nurse-led programme on comorbidity management and impact of a patient selfassessment of disease activity on the management of rheumatoid arthritis: results of a prospective, multicentre, randomised, controlled trial (COMEDRA). Ann Rheum. Dis. 2015; 74:1725-33.

6. Silva TC, Dantas AB, Menezes BD, Silveira EVS, Reis HG, Silveira JP, et al.Método de adesão ao tratamento de pacientes hipertensos. Arch Health Invest. 2017; 6(4):155-158.

7. Coutinho AT, Popim RC, Carregã K, Spiri WC. Integralidade do cuidado com o idoso na Estratégia de Saúde da Família: visão da equipe. Esc. Anna Nery. 2013; 17(4):628-37.

8. Souza NPG, Oliveira GYM, Girão ALA, Souza LM, Maniva SJCF, Freitas CHA. Adoecimento por hipertensão arterial e diabetes mellitus: concepções de um grupo de pacientes hospitalizados. Rev. enferm. UERJ. 2015; 23(1):52-7.

9. Hanus JS, Simões PW, Amboni G, Ceretta LB, Tuon LGB. Associação entre a qualidade de vida e adesão à medicação de indivíduos hipertensos. Acta Paul. Enferm. (Online). 2014; 28(4):381-7.

10. Tavares DMS, Guimarães MO, Ferreira PCS, Dias FA, Martins NPF, Rodrigues LR. Qualidade de vida e adesão ao tratamento farmacológico entre idosos hipertensos. Rev. bras. enferm. (Online) 2016; 69(1):134-41.

11. Costa YF, Araújo OC, Almeida LBM, Viegas SMF. O papel educativo do enfermeiro na adesão ao tratamento da Hipertensão Arterial Sistêmica: revisão integrativa da literatura. O Mundo da Saúde. 2014; 38(4):473-81.

12. Wang W, Lau Y, Chow A, Thompson DR, He HG. Health-related quality of life and social support among Chinese patients with coronary heart disease in mainland China. European Journal of Cardiovascular Nursing. 2014; 13(1):48-54.

13. Santos FPA, Nery AA, Matumoto S. A produção do cuidado a usuários com hipertensão arterial e as tecnologias em saúde. Esc. Enferm. USP. 2013; 47(1):107-14.

14. Guedes MBOG, Lima KC, Caldas CP, Veras RP. Apoio social e o cuidado integral à saúde do idoso. Physis Revista de Saúde Coletiva. 2017; 27940:1185-1204

15. Almeida RT, Ciosak SI. Comunicação do idoso e equipe de Saúde da Família: há integralidade?. Rev. latinoam. enferm. (Online). 2013; 21(4):1-7.

16. Pocock SJ. Clinical trials-a practical approach. New York: John Wiley \& Sons; 1989.
17. Morisky DE, Green LW, Levine DM. Concurrent and predective validity of self-reported measure of medication adherence. Med. Care. 1986; 24(1):67-74.

18. Sewitch MJ, Abrahamowicz M, Barkun A, Bitton A, Wild GE, Cohen $A$, et al. Patient nonadherence to medication in inflammatory Bowel disease. Am J Gastroenterol. 2003; 98(7):1535-44. 19. Starfield B. Atenção primária: equilíbrio entre necessidades de saúde, serviços e tecnologia. Brasília: Unesco, MS, 2002.

20.Sala A, Luppi CG, Simões O, Marsiglia RG. Integralidade e Atenção Primária à Saúde: avaliação na perspectiva dos usuários de unidades de saúde do município de São Paulo. Saúde Soc. São Paulo. 2011; 20(4):948-60.

21. Carvalho AKM, Leopoldino RWD, Silva JEG, Cunha CP. Adesão ao tratamento medicamentoso em usuários cadastrados no Programa Hiperdia no município de Teresina (PI). Ciência \& Saúde Coletiva. 2012; 17(7):1885-92.

22. Silva LFRS, Marino JMR, Guidoni CM, Girotto E. Fatores associados à adesão ao tratamento anti-hipertensivo por idosos na atenção primária. Rev. Ciênc. Farm. Básica Apl. 2014; 35(2):269-76. 23. Nascimento ACG, Alves ACP, Almeida AIM, Oliveira CJ. Características da adesão terapêutica em pessoas com hipertensão arterial e identificação do diagnóstico de enfermagem "falta de adesão" na atenção primária. Rev. APS. 2013; 16(4):365-77.

24. Schmieder R, Gitt AK, Koch C, Bramlage P, Ouarrak T, Tschõpe $D$, et al. Achievement of individualized treatment targets in patients with comorbid type- 2 diabetes and hypertension: 6 months results of the dialogue registry. BMC Endocrine Disorders. 2015; 15(23):2-12.

25. Machado LM, Colomé JS, Silva RM, Sangoi TP, Freitas NQ. Significados do fazer profissional na estratégia de saúde da família: atenção básica enquanto cenário de atuação. J Rev Fundam Care Online. 2016; 8(1):4026-35.

26. Lima LL, Moreira TMM, Jorge MSB. Produção do cuidado a pessoas com hipertensão arterial: acolhimento, vínculo e corresponsabilização. Rev. bras. enferm. (Online). 2013; 66(4):514-22. 27. Faquinello P, Marcon SS. Amigos e vizinhos: uma rede social ativa para adultos e idosos hipertensos. Esc. Enferm. USP. 2012; 45(6):1345-52

28. Silva-Costa A, Griep RH, Rotenberg L. Disentangling the effects of insomnia and night work on cardiovascular diseases: a study in nursing professionals. Braz J Med Biol Res. 2015; 48(2):120-7. 29. Santos CKR, Moraes JRMM, Santos NLP, Souza TV, Morais RCM, Azevedo SD. Qualidade da assistência de enfermagem em uma emergência pediátrica: perspectiva do acompanhante. Rev. enferm. UERJ. 2016; 24(4):e17560. 